\title{
A Bayesian Approach to Respiration Rate Estimation via Pulse-Based Ultra-Wideband Signals
}

\author{
Invited Paper \\ Hamza Soğancı, Sinan Gezici, and Orhan Arıkan \\ Dept. of Electrical and Electronics Engineering, Bilkent University, Bilkent, Ankara 06800, Turkey \\ \{hsoganci, gezici, oarikan\}@ee.bilkent.edu.tr
}

\begin{abstract}
In this paper, theoretical limits on estimation of respiration rates via pulse-based ultra-wideband (UWB) signals are studied in the presence of prior information about respiration related signal parameters. First, a generalized Cramer-Rao lower bound (G-CRLB) expression is derived, and then simplified versions of the bound are obtained for sinusoidal displacement functions. In addition to the derivation of the theoretical limits, a two-step suboptimal estimator based on matched filter (correlation) processing and maximum a posteriori probability (MAP) estimation is proposed. It is shown that the proposed estimator performs very closely to the theoretical limits under certain conditions. Simulation results are presented to investigate the theoretical results.
\end{abstract}

Index Terms-Ultra-wideband (UWB), generalized CramerRao lower bound (G-CRLB), maximum a posteriori probability (MAP) estimation.

\section{INTRODUCTION}

The large bandwidth of ultra-wideband (UWB) signals facilitates various applications such as high data rate communications [1] and accurate position estimation [2]. Also, UWB signals can be used to detect movements and to estimate movement related parameters in radar-type applications [3][9]. Due to the high time resolution of UWB signals, even small movements such as chest-cavity displacements of a human can be detected, which can be used in the estimation of vital signal parameters. Estimation of vital signal parameters is important in many scenarios, including searching people under debris after an earthquake, through-the-wall health monitoring of hostages, and non-invasive patient monitoring [7].

In [4], various medical applications of UWB signals are presented, and their penetration and reflection properties are investigated. The channel characteristics of signals reflecting from a human chest are studied in [8], which also proposes an algorithm for respiration rate estimation. In [9], respiratory detection of hidden humans via UWB signals is implemented. A mathematical framework for estimation of vital signal parameters is established in [7], which employs the Fourier transform and motions filters for estimation of respiration and heartbeat rates.

The theoretical limits on estimation of respiration rates are studied in [5] and Cramer-Rao lower bound (CRLB) expressions are obtained. [6] extends that study to multipath channels and derives theoretical limits and suboptimal estimation algorithms. Although the theoretical limits on estimation of respiration rates are obtained in [5] and [6], no studies have considered respiration rate estimation via UWB signals in the Bayesian framework [10]; that is, in the presence

\footnotetext{
${ }^{0}$ The authors wish to acknowledge the activity of the Network of Excellence in Wireless COMmunications NEWCOM++ of the European Commission (contract n. 216715) that motivated this work.
}

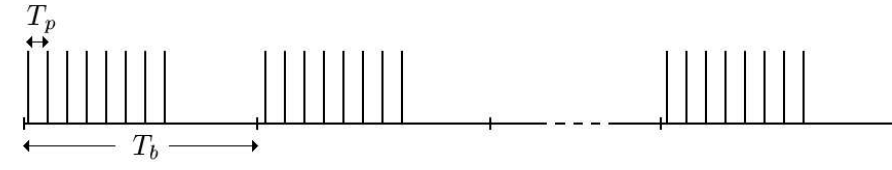

Fig. 1. Transmitted signal structure for respiration rate estimation.

of prior statistical information about respiration related parameters. Since it is possible to obtain such information in practice from respiration rate measurements, it is important to obtain theoretical lower bounds and practical estimators in the presence of such prior information. This paper provides a framework for estimation of respiration rates via pulsebased UWB signals in the presence of prior information by deriving generalized CRLB (G-CRLB) expressions and proposing a practical estimator based on matched filter (correlation) processing and maximum a posteriori probability (MAP) estimation. Although the estimation of respiration rates is considered, the ideas in this paper can also be extended to estimation of other periodic movements via UWB pulses.

\section{SignAl MODEL}

In order to estimate the respiration rate, a sequence of pulse bursts is transmitted towards the subject and the reflections are collected and processed. The transmitted signal structure is shown in Fig. 1, which is expressed as

$$
s(t)=\frac{1}{\sqrt{N}} \sum_{k=0}^{N-1} w\left(t-k T_{\mathrm{b}}\right),
$$

where $N$ is the number of bursts, $T_{\mathrm{b}}$ is the burst period, and $w(t)$ is a burst of pulses. Each burst comprises of $M$ pulses and is given by

$$
w(t)=\sum_{j=0}^{M-1} p\left(t-j T_{\mathrm{p}}\right),
$$

with $p(t)$ denoting the transmitted UWB pulse and $T_{\mathrm{p}}$ being the interval between consecutive pulses. It is assumed that $T_{\mathrm{p}}>T_{\mathrm{w}}$ where $T_{\mathrm{w}}$ denotes the width of $p(t)$.

The main reason for using the signal structure in (1) is that pulses in each burst are employed to obtain a reliable channel profile (i.e., to improve the signal-to-noise ratio (SNR)), and comparison of channel profiles obtained from consecutive bursts is used to estimate certain parameters (e.g., respiration rate) of the subject in the environment.

In this paper, an additive white Gaussian noise (AWGN) channel with a single path component is considered, which 
results in the following received signal:

$$
r(t)=g_{\boldsymbol{\theta}}(t)+\sigma n(t)
$$

where $n(t)$ denotes zero-mean white Gaussian noise with unit spectral density, and

$$
g_{\boldsymbol{\theta}}(t)=\frac{1}{\sqrt{N}} \sum_{k=0}^{N-1} w\left(t-k T_{\mathrm{b}}-h_{k}(\boldsymbol{\theta})\right),
$$

with $h_{k}(\boldsymbol{\theta})$ representing the periodic displacement function induced by the respiration of the subject being monitored [5]-[7], and $\boldsymbol{\theta}=\left[\theta_{1} \cdots \theta_{K}\right]$ denoting the unknown signal parameters. It is assumed that the range of the displacement function and the pulse $p(t)$ satisfy $(M-1) T_{\mathrm{p}}+T_{\mathrm{w}}+$ $\max \left\{h_{k}(\boldsymbol{\theta})\right\}-\min \left\{h_{k}(\boldsymbol{\theta})\right\}<T_{\mathrm{b}}$, so that there is no overlap between consecutive pulse bursts, which is usually the case in practical situations.

Although the single path model in (3) is not very realistic for wideband pulse-based systems, it is an important first step towards understanding of a real system since the main ideas in the analysis can be extended to multipath scenarios as in [6]. The main purpose of this study is to illustrate how the prior information about the respiration related parameters can be incorporated into the theoretical limits and practical estimators. In addition, the model in (3) gets more accurate when directional antennas are used for transmission and reception, and/or an efficient clutter removal algorithms are employed before parameter estimation [6].

\section{TheORETICAL Limits}

If the signal $r(t)$ in (3) is observed over the time interval $[0, T]$, with $T=N T_{\mathrm{b}}$, the $\log$-likelihood function of $\boldsymbol{\theta}$ is given by [5], [11]

$$
\Lambda(\boldsymbol{\theta})=c-\frac{1}{2 \sigma^{2}} \int_{0}^{T}\left[r(t)-g_{\boldsymbol{\theta}}(t)\right]^{2} d t,
$$

where $c$ denotes a constant that is independent of $\boldsymbol{\theta}$.

In the presence of prior information about the unknown parameter vector $\boldsymbol{\theta}$, the G-CRLB is expressed as [10]

$$
\mathrm{E}\left\{(\hat{\boldsymbol{\theta}}-\boldsymbol{\theta})(\hat{\boldsymbol{\theta}}-\boldsymbol{\theta})^{T}\right\} \geq \mathbf{I}^{-1},
$$

where $\hat{\boldsymbol{\theta}}$ is an unbiased estimate of $\boldsymbol{\theta}$, and $\mathbf{I}$ is the information matrix given by

$$
\mathbf{I}=\mathbf{I}_{\mathrm{D}}+\mathbf{I}_{\mathrm{P}},
$$

with $\mathbf{I}_{D}$ and $\mathbf{I}_{\mathrm{P}}$ representing the information matrix obtained from the data (observations) and from the prior knowledge, respectively. The matrices in (7) are given by ${ }^{1}$

$$
\left[\mathbf{I}_{\mathrm{D}}\right]_{i j}=\mathrm{E}\left\{\frac{\partial \Lambda(\boldsymbol{\theta})}{\partial \theta_{i}} \frac{\partial \Lambda(\boldsymbol{\theta})}{\partial \theta_{j}}\right\},
$$

where the expectation is over both the received signal and the $\boldsymbol{\theta}$ parameter, and

$$
\left[\mathbf{I}_{\mathrm{P}}\right]_{i j}=\mathrm{E}\left\{\frac{\partial \log \pi(\boldsymbol{\theta})}{\partial \theta_{i}} \frac{\partial \log \pi(\boldsymbol{\theta})}{\partial \theta_{j}}\right\}
$$

where $\pi(\boldsymbol{\theta})$ represents the probability density function for the parameter of interest $\boldsymbol{\theta}$.

\footnotetext{
${ }^{1}[\mathbf{B}]_{i j}$ denotes the element of matrix $\mathbf{B}$ in the $i$ th row and $j$ th column.
}

In order to obtain the G-CRLB in (6), we first need to calculate $\mathbf{I}_{\mathrm{D}}$ in (8). From (4) and (5), it can be expressed, after some manipulation, as

$$
\left[\mathbf{I}_{\mathrm{D}}\right]_{i j}=\frac{\tilde{E}}{N \sigma^{2}} \sum_{k=0}^{N-1} \mathrm{E}\left\{\frac{\partial h_{k}(\boldsymbol{\theta})}{\partial \theta_{i}} \frac{\partial h_{k}(\boldsymbol{\theta})}{\partial \theta_{j}}\right\},
$$

where the expectation is now only over $\boldsymbol{\theta}$, and $\tilde{E}$ is the energy of the first derivative of the pulse burst $w(t)$, i.e.,

$$
\tilde{E}=\int_{-\infty}^{\infty}\left(\frac{d w(t)}{d t}\right)^{2} d t=M \int_{-\infty}^{\infty}\left(\frac{d p(t)}{d t}\right)^{2} d t .
$$

The information matrix $\mathbf{I}_{P}$ in (9) due to the prior information can easily be calculated for a given probability distribution. In the special case of independent parameters $\theta_{1}, \ldots, \theta_{K},(9)$ simplifies to

$$
\left[\mathbf{I}_{\mathrm{P}}\right]_{i j}=\mathrm{E}\left\{\frac{\partial \log \pi_{i}\left(\theta_{i}\right)}{\partial \theta_{i}} \frac{\partial \log \pi_{j}\left(\theta_{j}\right)}{\partial \theta_{j}}\right\},
$$

where $\pi_{i}(x)$ is the probability density function for parameter $\theta_{i}$.

Let $\theta_{1}=f$ denote the respiration rate parameter to be estimated. Then, the G-CRLB for estimating $f$ can be stated as

$$
\operatorname{Var}\{\hat{f}\} \geq\left[\mathbf{I}^{-1}\right]_{11}
$$

where I is given by (7), (9) and (10).

\section{A. Sinusoidal Displacement Function}

Although the theoretical upper bounds on the variances of unbiased respiration rate estimators can be obtained from the generic expression in (13), simpler expressions can be obtained for specific models related to respiration. Commonly, the displacement function $h_{k}(\boldsymbol{\theta})$ can be modeled to have three unknown parameters; frequency $f$, phase $\phi$ and time shift $A$ corresponding to the maximum displacement from a nominal position [5]. In other words, the unknown parameter vector can be defined as $\boldsymbol{\theta}=\left[\begin{array}{ll}f \phi A & \text {, where } f \text { represents }\end{array}\right.$ the main parameter of interest, i.e., the respiration rate. The phase parameter is another unknown as the initial position of the object (i.e., chest cavity) is not known by the receiver. In addition, the time shift corresponding to the maximum displacement amount from the nominal object position, $A$, is usually unknown.

Prior distributions of parameters $f$ and $A$ can be available from previous measurements of respiration rates. However, the phase parameter can be modeled to be completely random. Therefore, for a given joint probability distribution of $f$ and $A$, the information matrix $\mathbf{I}_{\mathrm{P}}$ in (9) due to the prior knowledge can be represented as

$$
\mathbf{I}_{\mathrm{P}}=\left[\begin{array}{ccc}
\gamma_{11} & 0 & \gamma_{13} \\
0 & 0 & 0 \\
\gamma_{13} & 0 & \gamma_{33}
\end{array}\right]
$$

where it is assumed that no prior information is available about the phase parameter.

For the special case of a displacement function modeled by a sinusoidal function [6], [7], $h_{k}(\boldsymbol{\theta})$ is expressed as

$$
h_{k}(\boldsymbol{\theta})=A \sin \left(2 \pi f k T_{\mathrm{b}}+\phi\right) .
$$


Then, a closed-form expression for the theoretical limit can be obtained as in the following proposition.

Proposition 1: Consider the information matrix in (14) related to the prior knowledge and the displacement function in (15). Then, the G-CRLB for unbiased estimators of $f$ is expressed as

$$
\operatorname{Var}\{\hat{f}\} \geq\left(\gamma_{11}-\frac{2 \sigma^{2} \gamma_{13}^{2}}{\tilde{E}+2 \sigma^{2} \gamma_{33}}+\frac{\tilde{E} \alpha_{A} \pi^{2} T_{\mathrm{b}}^{2}\left(N^{2}-1\right)}{6 \sigma^{2}}\right)^{-1}
$$

where $\alpha_{A} \doteq \mathrm{E}\left\{A^{2}\right\}$.

Proof: Please see Appendix A.

Proposition 1 states that as the prior information on $f(A)$, represented by $\gamma_{11}\left(\gamma_{33}\right)$, or the second moment of $A, \alpha_{A}$, increases, the G-CRLB decreases. This is expected since more prior information related to the respiration parameters and larger movements of the chest (i.e., larger $A$ values) facilitate better respiration rate estimation. In addition, the G-CRLB also decreases when the burst duration, the number of bursts, or $\tilde{E} / \sigma^{2}$ increases.

The following corollary considers the special case in which $\gamma_{13}=0$ in (14).

Corollary 1: Consider the information matrix in (14) related to the prior knowledge and the displacement function in (15), and assume that $\gamma_{13}=0$. Then, the G-CRLB for unbiased estimators of $f$ is given by

$$
\operatorname{Var}\{\hat{f}\} \geq\left(\gamma_{11}+\frac{\tilde{E} \alpha_{A} \pi^{2} T_{\mathrm{b}}^{2}\left(N^{2}-1\right)}{6 \sigma^{2}}\right)^{-1}
$$

where $\alpha_{A} \doteq \mathrm{E}\left\{A^{2}\right\}$.

Corollary 1 implies that for $\gamma_{13}=0$, the prior information related to $A$ does not improve the theoretical lower bound. In this case, the G-CRLB decreases only when $\gamma_{11}$, the second moment of $A$ (i.e., $\alpha_{A}$ ), the burst duration, the number of bursts, and/or $\tilde{E} / \sigma^{2}$ increases. Note that the bound depends on the probability distribution of $A$ only through $\alpha_{A}$; that is, as long as the second moment of $A$ is fixed, the probability distribution of $A$ does not affect the G-CRLB for $\gamma_{13}=0$.

Note that the G-CRLB expression in (16) is generic in the sense that it does not assume any specific probability distributions for $f$ and $A$. For the special case of a jointly Gaussian distribution for $\left[\begin{array}{ll}f & A\end{array}\right]^{T}$, more specific expressions can be obtained. Let

$$
\left[\begin{array}{l}
f \\
A
\end{array}\right] \sim \mathcal{N}\left(\left[\begin{array}{l}
\mu_{f} \\
\mu_{A}
\end{array}\right],\left[\begin{array}{cc}
\sigma_{f}^{2} & \rho_{f A} \\
\rho_{f A} & \sigma_{A}^{2}
\end{array}\right]\right) .
$$

Then, $\gamma_{11}, \gamma_{33}$ and $\gamma_{13}$ in (16) can be obtained from (9) as

$$
\gamma_{11}=\sigma_{A}^{2} / \eta, \quad \gamma_{33}=\sigma_{f}^{2} / \eta, \quad \gamma_{13}=-\rho_{f A} / \eta,
$$

where $\eta \doteq \sigma_{f}^{2} \sigma_{A}^{2}-\rho_{f A}^{2}$.

Note that for $\rho_{f A}=0$; i.e., when $f$ and $A$ are uncorrelated, the G-CRLB expression in (16) becomes

$$
\operatorname{Var}\{\hat{f}\} \geq\left(\frac{1}{\sigma_{f}^{2}}+\frac{\tilde{E} \alpha_{A} \pi^{2} T_{\mathrm{b}}^{2}\left(N^{2}-1\right)}{6 \sigma^{2}}\right)^{-1} .
$$

In addition, as $\sigma_{f}^{2} \rightarrow \infty$, the bound converges to $6 \sigma^{2} /\left(\tilde{E} \alpha_{A} \pi^{2} T_{\mathrm{b}}^{2}\left(N^{2}-1\right)\right)$, which is the same as the expression in [5] (c.f. (18)) when $\alpha_{A}=\mathrm{E}\left\{A^{2}\right\}$ is replaced by $A^{2}$

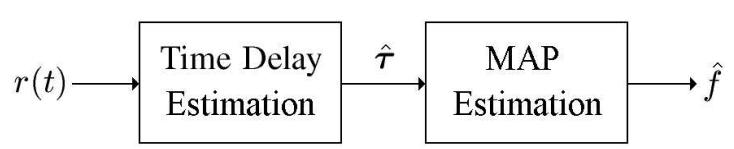

Fig. 2. A two-step suboptimal solution for respiration rate estimation.

(i.e., when $A$ is deterministic). In other words, as the amount of prior information on $f$ converges to zero, the G-CRLB converges to the CRLB as expected.

\section{Suboptimal Estimator}

As discussed in [6], optimal estimation of respiration rate based directly on $r(t)$ has high computational complexity. In this section, a suboptimal estimator is proposed in order to perform closely to the theoretical limits obtained in the previous section with low complexity. The proposed estimator has a two-step structure as shown in Fig. 2. In the first step, the time delay of each burst is estimated from the $M$ pulses in the burst. Let $\tau_{k}$ denote the delay of the $k$ th burst for $k=0,1, \ldots, N-1$. Note that each $\tau_{k}$ can be estimated by the conventional timeof-arrival (TOA) estimation algorithm based on matched filter (or, correlator) outputs [2]. The time delay estimates obtained in the first step, namely, $\hat{\tau}=\left[\hat{\tau}_{0} \hat{\tau}_{1} \cdots \hat{\tau}_{N-1}\right]$, are used by a MAP estimator [11] in the second step in order to estimate the unknown parameters.

It is shown in [12] that time delay estimates obtained from matched filter outputs can be modeled as Gaussian random variables around the true time delay under certain conditions. Specifically, for following the signal model

$$
\tilde{r}(t)=\frac{1}{\sqrt{N}} w\left(t-\tau_{k}\right)+\sigma n(t), \quad t \in\left[0, T_{\mathrm{b}}\right],
$$

where $n(t)$ is zero mean white Gaussian noise with unit spectral density, and $\tau_{k}$ is the time delay to be estimated, if $\tau_{k}$ is estimated by matched-filter outputs with large SNR $\beta^{2}$ values, ${ }^{2}$ the time delay estimate can be modeled as

$$
\hat{\tau}_{k}=\tau_{k}+n_{k}
$$

with $n_{k} \sim \mathcal{N}\left(0, \sigma_{0}^{2}\right)$ and $\sigma_{0}^{2}=1 /\left(4 \pi^{2} \beta^{2} \mathrm{SNR}\right)$ [5], [13].

This result implies that the time delay estimates obtained in the first step of the proposed algorithm in Fig. 2 can be modeled to have zero mean Gaussian errors for large SNR $\beta^{2}$ values. Note that in the proposed algorithm, $r(t)$ for $t \in\left[(k-1) T_{\mathrm{b}}, k T_{\mathrm{b}}\right]$ is used for estimating $\tau_{k}$ using a conventional matched-filter approach for $k=0,1, \ldots, N-1$. Therefore, for large SNR $\beta^{2}$, these time delay estimates can be modeled as

$$
\hat{\tau}_{k}=h_{k}(\boldsymbol{\theta})+n_{k},
$$

for $k=0,1, \ldots, N-1$, with $n_{k} \sim \mathcal{N}\left(0, \sigma_{0}^{2}\right)$. Since each time delay estimate is obtained from a different portion of the signal, $n_{0}, n_{1}, \ldots, n_{N-1}$ are independent.

Based on the observations in the preceding paragraphs, the MAP estimator in Fig. 2 calculates the unknown parameter

\footnotetext{
${ }^{2} \beta$ is the effective bandwidth, which is defined by $\beta^{2}=$ $\int f^{2}|W(f)|^{2} d f / \int|W(f)|^{2} d f$, where $W(f)$ is the Fourier transform of $w(t)$.
} 
vector $\boldsymbol{\theta}$ as follows:

$$
\begin{aligned}
& \hat{\boldsymbol{\theta}}=\arg \max _{\boldsymbol{\theta}}\{\log p(\hat{\boldsymbol{\tau}} \mid \boldsymbol{\theta})+\log \pi(\boldsymbol{\theta})\} \\
& =\arg \min _{\boldsymbol{\theta}}\left\{\frac{1}{2 \sigma_{0}^{2}} \sum_{k=0}^{N-1}\left(\hat{\tau}_{k}-h_{k}(\boldsymbol{\theta})\right)^{2}-\log \pi(\boldsymbol{\theta})\right\} .
\end{aligned}
$$

Note that the conditional distribution of the time delay estimates, $p(\hat{\boldsymbol{\tau}} \mid \boldsymbol{\theta})$, is modeled according to (23).

In the case of Gaussian priors as in (18), the MAP estimator in $(25)$ becomes

$$
\begin{aligned}
\hat{\boldsymbol{\theta}}= & \arg \min _{\boldsymbol{\theta}}\left\{\frac{1}{\sigma_{0}^{2}} \sum_{k=0}^{N-1}\left(\hat{\tau}_{k}-h_{k}(\boldsymbol{\theta})\right)^{2}+\frac{1}{\eta}\left[\sigma_{A}^{2}\left(f-\mu_{f}\right)^{2}\right.\right. \\
& \left.\left.-2 \rho_{f A}\left(f-\mu_{f}\right)\left(A-\mu_{A}\right)+\sigma_{f}^{2}\left(A-\mu_{A}\right)^{2}\right]\right\},
\end{aligned}
$$

where $\eta=\sigma_{f}^{2} \sigma_{A}^{2}-\rho_{f A}^{2}$.

Proposition 2 states the asymptotic optimality property of the proposed two-step estimator.

Proposition 2: For a given set of time delay measurements $\hat{\tau}=\left[\hat{\tau}_{0} \hat{\tau}_{1} \cdots \hat{\tau}_{N-1}\right]$ modeled by (23), the G-CRLB for the covariance matrix of an unbiased estimate of $\boldsymbol{\theta}$ is the same as the expression given by (6)-(10).

Proof: Please see Appendix B.

In other words, Proposition 2 states that the theoretical limits on the estimation of the respiration rate parameter based on the measurements in (23) and based on $r(t)$ in (3) are the same. Since the MAP estimator is known to achieve the G-CRLB asymptotically [10], the proposed estimator can achieve the theoretical limit of the original problem as well under certain conditions.

\section{Simulation Results}

In this section, numerical evaluations and simulations are performed in order to evaluate the G-CRLB expressions derived in Section III, and to investigate the performance of the proposed suboptimal estimator in the previous section.

For the pulse shape in (2), the second derivative of the Gaussian pulse is used, which is given by [2]

$$
p(t)=\left(1-\frac{4 \pi t^{2}}{\zeta^{2}}\right) e^{-\frac{2 \pi t^{2}}{\zeta^{2}}} / \sqrt{E_{\mathrm{p}}},
$$

where $E_{\mathrm{p}}$ is used to adjust the energy of the pulse in the simulations, and $\zeta$ determines the pulse width $\left(T_{\mathrm{w}} \approx 2.5 \zeta\right)$.

For the following results, $N=50$ bursts and $T_{\mathrm{b}}=0.1$ second are used. In addition, the displacement function in (15) is considered, and the prior distribution of $f$ and $A$ is assumed to be jointly Gaussian according to (18) with $\mu_{f}=0.5 \mathrm{~Hz}$, $\mu_{A}=0.1 \mathrm{~ns}, \sigma_{f}=0.1 \mathrm{~Hz}$, and $\sigma_{A}=0.02 \mathrm{~ns}\left(\rho_{f A}\right.$ is specified below).

In Fig. 3 and Fig. 4, the G-CRLB expression in (16) is plotted against the SNR for various pulse widths for $\rho_{f A}=0$ and $\rho_{f A}=-0.2$, respectively. ${ }^{3}$ It is observed from the figures that the accuracy increases as the pulse width is decreased. This is intuitive as higher time resolution results in better localization of the chest cavity. In addition, larger correlations

\footnotetext{
${ }^{3}$ The square-roots of the results are plotted and the lower bounds are obtained in the unit of $\mathrm{Hz}$.
}

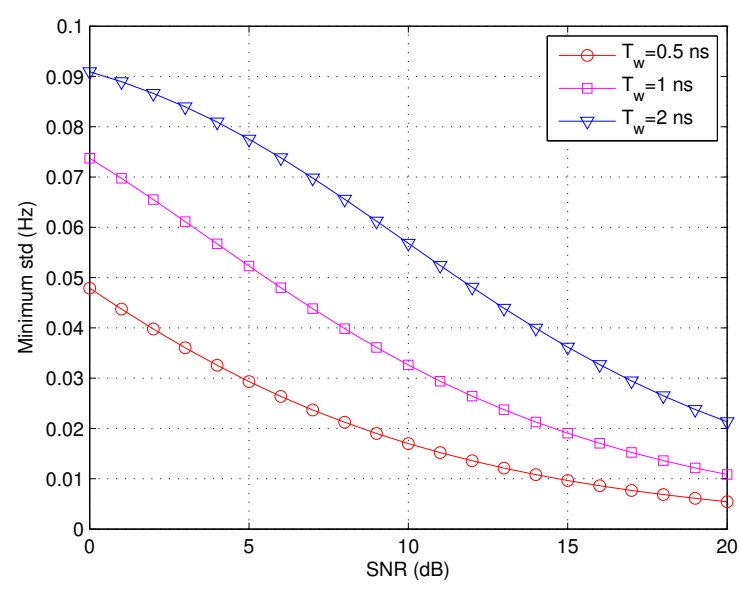

Fig. 3. G-CRLB versus SNR for various pulse widths, where $\rho_{f A}=0$ and $N=50$ bursts are transmitted.

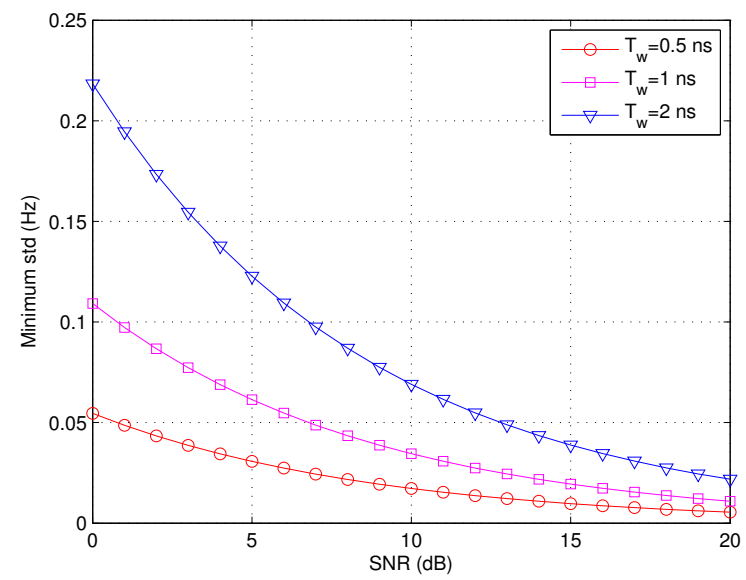

Fig. 4. G-CRLB versus SNR for various pulse widths, where $\rho_{f A}=-0.2$ and $N=50$.

between $f$ and $A$ result in worse accuracy (i.e., larger GCRLBs). However, as the pulse width is decreased, the effects of correlations on the accuracy decrease significantly.

In Fig. 5, the G-CRLB is plotted versus the pulse width for various SNRs for $\rho_{f A}=0$. Again it is observed that the accuracy increases as the pulse width is decreased. It is also noted that at high SNRs, the second term in (17) becomes the dominant factor in determining the theoretical limit, which results in a linear relation between $T_{\mathrm{w}}$ and the square-root of the theoretical limit. However, at low SNRs, the first term in (17) becomes significant as well; hence, the relation becomes non-linear as shown by the plot for $\mathrm{SNR}=0 \mathrm{~dB}$.

Finally, Fig. 6 compares the performance of the suboptimal MAP estimator in Section IV with the G-CRLB for $T_{\mathrm{w}}=1 \mathrm{~ns}$ and $\rho_{f A}=0$. It is observed that the performance of the MAP estimator gets close to the theoretical limits at high SNRs as expected.

\section{CONCLUding REMARKS}

In this paper, theoretical limits on respiration rate estimation via pulse-based UWB signals have been studied, and closedform expressions for the G-CRLB have been obtained. In addition, a two-step suboptimal estimator has been proposed and 


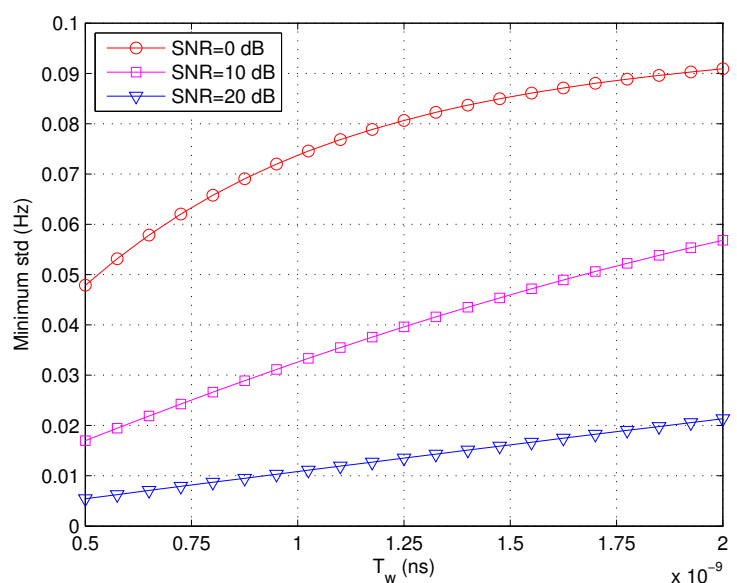

Fig. 5. G-CRLB versus pulse width for various SNRs, where $\rho_{f A}=0$ and $N=50$.

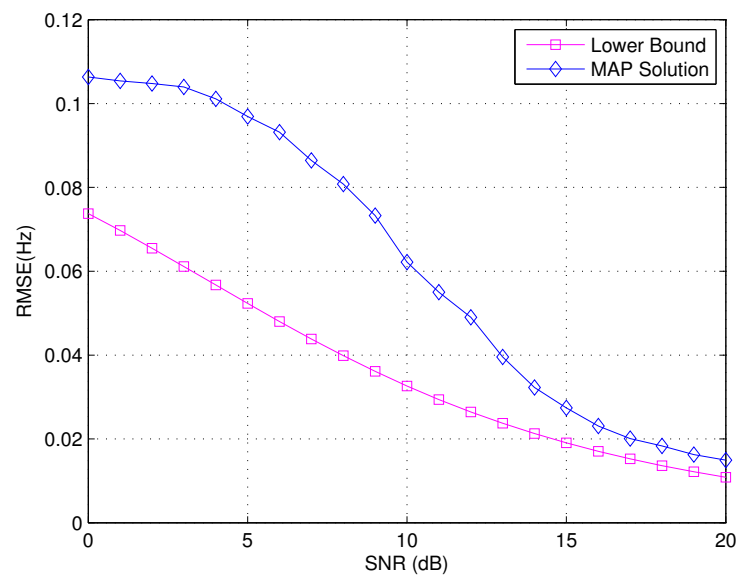

Fig. 6. RMSE versus SNR for the MAP estimator and the G-CRLB for $T_{\mathrm{w}}=1 \mathrm{~ns}, \rho_{f A}=0$ and $N=50$.

its asymptotic optimality property has been shown. Although this study considers the estimation of respiration rates, the ideas can also be extended to estimation of other periodic movements via UWB pulses.

\section{APPENDIX}

\section{A. Proof of Proposition 1}

First, the expectation term in (10) can be evaluated from (15) as

$$
\begin{aligned}
& \mathrm{E}\left\{\left(\frac{\partial h_{k}(\boldsymbol{\theta})}{\partial f}\right)^{2}\right\}=2 \pi^{2} k^{2} T_{b}^{2} \mathrm{E}\left\{A^{2}\right\}, \\
& \mathrm{E}\left\{\left(\frac{\partial h_{k}(\boldsymbol{\theta})}{\partial \phi}\right)^{2}\right\}=\frac{\mathrm{E}\left\{A^{2}\right\}}{2}, \mathrm{E}\left\{\left(\frac{\partial h_{k}(\boldsymbol{\theta})}{\partial A}\right)^{2}\right\}=\frac{1}{2}, \\
& \mathrm{E}\left\{\frac{\partial h_{k}(\boldsymbol{\theta})}{\partial f} \frac{\partial h_{k}(\boldsymbol{\theta})}{\partial \phi}\right\}=\pi k T_{b} \mathrm{E}\left\{A^{2}\right\}, \\
& \mathrm{E}\left\{\frac{\partial h_{k}(\boldsymbol{\theta})}{\partial f} \frac{\partial h_{k}(\boldsymbol{\theta})}{\partial A}\right\}=\mathrm{E}\left\{\frac{\partial h_{k}(\boldsymbol{\theta})}{\partial \phi} \frac{\partial h_{k}(\boldsymbol{\theta})}{\partial A}\right\}=0,
\end{aligned}
$$

where the complete randomness of $\phi$ is modeled as $\bmod (\phi, 2 \pi) \sim \mathcal{U}[0,2 \pi)$, where $\bmod (\phi, 2 \pi)$ represents a modulo- $2 \pi$ operation on $\phi$, and $\mathcal{U}$ denotes a uniform distribution. Then, the results in (28) can be inserted into (10), and $\mathbf{I}_{\mathrm{D}}$ can be obtained. Finally, that $\mathbf{I}_{\mathrm{D}}$ expression and $\mathbf{I}_{\mathrm{P}}$ in (14) can be added, and the first element of the inverse of $\mathbf{I}_{\mathrm{D}}+\mathbf{I}_{\mathrm{P}}$ can be calculated after some manipulation, which yields the result in the proposition.

\section{B. Proof of Proposition 2}

For the model in (23), the distribution of $\hat{\tau}$ for a given values of $\boldsymbol{\theta}$ is expressed as

$$
p_{\boldsymbol{\theta}}(\hat{\boldsymbol{\tau}})=\frac{1}{\left(\sqrt{2 \pi} \sigma_{0}\right)^{N}} \exp \left\{-\frac{1}{2 \sigma_{0}^{2}} \sum_{k=0}^{N-1}\left(\hat{\tau}_{k}-h_{k}(\boldsymbol{\theta})\right)^{2}\right\} .
$$

Then, the formula for the information matrix related to the data,

$$
\left[\tilde{\mathbf{I}}_{\mathrm{D}}\right]_{i j}=\mathrm{E}\left\{\frac{\partial \log p_{\boldsymbol{\theta}}(\hat{\boldsymbol{\tau}})}{\partial \theta_{i}} \frac{\partial \log p_{\boldsymbol{\theta}}(\hat{\boldsymbol{\tau}})}{\partial \theta_{j}}\right\},
$$

can be used to obtain

$$
\left[\tilde{\mathbf{I}}_{\mathrm{D}}\right]_{i j}=\frac{1}{\sigma_{0}^{2}} \sum_{k=0}^{N-1} \mathrm{E}\left\{\frac{\partial h_{k}(\boldsymbol{\theta})}{\partial \theta_{i}} \frac{\partial h_{k}(\boldsymbol{\theta})}{\partial \theta_{j}}\right\} .
$$

The expression for $\sigma_{0}^{2}$ stated after (22), $\sigma_{0}^{2}=$ $1 /\left(4 \pi^{2} \beta^{2} \mathrm{SNR}\right)$, can be shown to be equal to $\sigma_{0}^{2}=N \sigma^{2} / \tilde{E}$. Therefore, (31) is equal to (10); i.e., $\tilde{\mathbf{I}}_{\mathrm{D}}=\mathbf{I}_{\mathrm{D}}$. In addition, since the information due to the prior distribution of $\boldsymbol{\theta}$ is the same in both scenarios, the total information matrix is the same in both cases.

\section{REFERENCES}

[1] H. Arslan, Z. N. Chen, and M.-G. Di Benedetto (editors), Ultra Wideband Wireless Communications, Wiley-Interscience, Oct. 2006.

[2] Z. Sahinoglu, S. Gezici, and I. Guvenc, Ultra-wideband Positioning Systems: Theoretical Limits, Ranging Algorithms, and Protocols, Cambridge University Press, 2008.

[3] J. D. Taylor (editor), Ultra-wideband Radar Technology, CRC Press, 2001.

[4] E. M. Staderini "UWB radars in medicine," IEEE Aerospace and Electronic Magazine, vol. 17, no. 1, pp. 13-18, Jan. 2002.

[5] S. Gezici and Z. Sahinoglu, "Theoretical limits for estimation of vital signal parameters using impulse radio UWB," IEEE International Conference on Communications, Glasgow, Scotland, June 24-27, 2007.

[6] S. Gezici, "Theoretical limits for estimation of periodic movements in pulse-based UWB systems," IEEE Journal of Selected Topics in Signal Processing, vol. 1, issue 3, pp. 405-417, Oct. 2007.

[7] S. Venkatesh, C. R. Anderson, N. V. Rivera, and R. M. Buehrer, "Implementation and analysis of respiration-rate estimation using impulsebased UWB," IEEE Military Communications Conference (MILCOM 2005), vol. 5, pp. 3314-3320, Atlantic City, NJ, Oct. 17-20, 2005.

[8] Y. Chen, et. al., "Non-invasive respiration rate estimation using ultrawideband distributed cognitive radar system," Proc. 28th IEEE EMBS Annual International Conference, pp. 920-923, Aug. 30-Sep. 3, 2006.

[9] G. Ossberger, et. al., "Non-invasive respiratory movement detection and monitoring of hidden humans using ultra wideband pulse radar,' IEEE International Workshop on Ultra Wideband Systems, pp. 395-399, Piscataway, NJ, May 18-21, 2004.

[10] Harry L. Van Trees, Detection, Estimation, and Modulation Theory, Part I, Wiley-Interscience, 1st edition, 2001.

[11] H. V. Poor, An Introduction to Signal Detection and Estimation, 2nd ed New York: Springer-Verlag, 1994.

[12] Y. Qi, "Wireless geolocation in a non-line-of-sight environment," Ph.D. dissertation, Princeton University, Dec. 2003.

[13] Y. Qi, H. Kobayashi, and H. Suda, "On time-of-arrival positioning in a multipath environment," IEEE Transactions on Vehicular Technology, vol. 55, no. 5, pp. 1516-1526, Sep. 2006. 